\title{
Combination transarterial chemoembolization and radiofrequency ablation therapy for early hepatocellular carcinoma
}

Myeong Jun Song ${ }^{1}$, Si Hyun Bae ${ }^{1}$, June Sung Lee ${ }^{2}$, Sung Won Lee ${ }^{1}$, Do Seon Song ${ }^{1}$, Chan Ran You ${ }^{1}$, Jong Young Choi ${ }^{1}$, and Seung Kew Yoon ${ }^{1}$

${ }^{1}$ Department of Internal Medicine, College of Medicine, The Catholic University of Korea, Seoul; ${ }^{2}$ Department of Internal Medicine, Inje University Ilsan Paik Hospital, Goyang, Korea

Received: April 26, 2014

Revised : August 13, 2014

Accepted: November 18, 2015

\section{Correspondence to}

\section{Chan Ran You, M.D.}

Department of Internal Medicine, College of Medicine, The Catholic University of Korea, 222 Ban-

po-daero, Seocho-gu, Seoul o6591, Korea

Tel: +82-2-2258-2073

Fax: +82-2-3481-4025

E-mail: ran_you@catholic.ac.kr
Background/Aims: We compared the recurrence of hepatocellular carcinoma (HCC) and the survival of patients who received radiofrequency ablation (RFA) after transarterial chemoembolization (TACE) with patients treated with TACE or RFA alone.

Methods: This study included 201 patients with HCC, who were consecutively enrolled at Seoul St. Mary's Hospital between December 2004 and February 2010. Inclusion criteria were a single $\mathrm{HCC} \leq 5.0 \mathrm{~cm}$ or up to three $\mathrm{HCCs} \leq 3.0 \mathrm{~cm}$. We used a propensity score model to compare HCC patients $(n=87)$ who received RFA after TACE (TACE + RFA) with those who received TACE $(n=71)$ or RFA alone $(n=$ 43).

Results: The median follow-up period was 33.3 months (range, 6.8 to 80.9). The TACE + RFA group showed significantly lower local recurrence than the RFA or TACE groups (hazard ratio [HR], 0.309; 95\% confidence interval [CI], 0.130 to 0.736 ; $p=0.008$; and HR, $0.352 ; 95 \% \mathrm{CI}, 0.158$ to $0.787 ; p=0.011$, respectively). The overall survival was significantly better in the TACE + RFA group compared to the RFA group (HR, $0.422 ; 95 \%$ CI, 0.185 to $0.964 ; p=0.041)$. However, the survival benefit was not different between the TACE + RFA and TACE groups $(p=0.124)$. Subgroup analysis showed that among patients with a tumor size $<3 \mathrm{~cm}$, the TACE + RFA group had significantly better long-term survival than those in the TACE or RFA groups $(p=0.017, p=0.004$, respectively).

Conclusions: TACE + RFA combination treatment showed favorable local recurrence and better overall survival rates in early-stage HCC patients. Patients with tumors $<3 \mathrm{~cm}$ are likely to benefit more from TACE + RFA combination treatment. Additional studies are needed for the selection of suitable HCC patients for TACE + RFA treatment.

Keywords: Radiofrequency ablation; Transarterial chemoembolization; Recurrence; Overall survival 


\section{INTRODUCTION}

Hepatocellular carcinoma (HCC) is the sixth most common cancer and third leading cause of cancer-related death with increasing incidence worldwide [1,2]. Despite the widespread use of surveillance programs in at-risk populations, more than half of HCC cases are diagnosed late, and curative therapies such as surgical resection, transplantation, or radiofrequency ablation (RFA) are possible in less than $30 \%$ of patients [3]. After curative treatment, patients face a very high risk of HCC recurrence in an underlying cirrhotic liver. Therefore, locoregional treatments are potentially useful alternative modalities for many patients. Although surgical resection is the gold standard treatment for early-stage HCC, many studies have reported that the efficacy of locoregional therapies is similar to that of resection [4-6].

Locoregional treatments for HCC, which include RFA, percutaneous ethanol injection, and transarterial chemoembolization (TACE), are promising modalities in patients with unresectable tumors. RFA and percutaneous ethanol injection are considered curative treatments for early-stage HCC [7-10]. TACE is the standard of care for the intermediate stages according to Barcelona Clinic for Liver Cancer (BCLC) staging and treatment [10]. Both RFA and TACE have shown overall survival (OS) benefits [11]. RFA has shown complete short-term necrosis in $\geq 90 \%$ of lesions smaller than $3 \mathrm{~cm}$ in diameter $[4,12,13]$. For lesions 3 to $5 \mathrm{~cm}$ in diameter, the percentage of complete necrosis varied between $50 \%$ and $70 \%$ [14]. Complete ablation was obtained in $<30 \%$ of lesions larger than $5 \mathrm{~cm}$ in diameter [14-16].

TACE is widely used to treat HCC patients who are not suitable candidates for curative treatments and bridge to liver transplantation $[10,17]$. The rationale for TACE is that intra-arterial chemotherapy with lipiodol and a chemotherapeutic agent followed by selective vascular embolization will result in a strong cytotoxic effect combined with ischemia [18,19]. In a previous study of transplant patients, selective/superselective TACE was more effective for tumors 3 to $5 \mathrm{~cm}$ in diameter [20].

Recent studies have reported that combining RFA with TACE to treat HCC may have a synergistic effect [21,22]. Pretreatment with TACE appears to increase the RFA zone and the probability of a complete response (CR). The reduction or elimination of blood flow after TACE increases the volume of ablation, because blood flow promotes heat loss due to RFA. Therefore, performing RFA after blocking the hepatic artery feeding the tumor is reasonable. The complication rate that resulted from the use of TACE before RFA was not more significant than using RFA alone.

Therefore, the synergistic effects of TACE and RFA might yield lower recurrence rates and better survival times than using TACE or RFA as monotherapies. We evaluated tumor recurrence and OS rates of early-stage HCC patients who received RFA after TACE compared to those treated with TACE or RFA alone.

\section{METHODS}

\section{Patients}

This was a retrospective cohort study conducted in a single center. The medical records of patients treated with TACE, followed by RFA or TACE as the initial treatment for HCC between December 2004 and February 2010 at Seoul St. Mary's Hospital, were reviewed. The diagnosis and HCC stage were assessed according to the American Association for the Study of Liver Disease guidelines and the BCLC staging classification [10]. Inclusion criteria were as follows: (1) a single $\mathrm{HCC} \leq 5 \mathrm{~cm}$ or up to three HCCs $\leq 3 \mathrm{~cm}$; (2) at least two HCC lesions of a minimum of $10 \mathrm{~mm}$ in diameter based on liver dynamic computed tomography (CT) and target lesions suitable for accurate repeated measurements [23]; (3) an Eastern Cooperative Oncology Group performance status of $\mathrm{O}$ or 1; (4) preserved liver function (Child-Pugh Class A or B); (5) no previous treatment for HCC; and (6) no vascular invasion. Exclusion criteria included another primary tumor, advanced liver disease (bilirubin levels $>3 \mathrm{mg} / \mathrm{dL}$ and aspartate aminotransferase [AST] or alanine transaminase [ALT] levels $>5 \times$ upper limit of normal), a thrombus within a main or branched portal vein, or extrahepatic metastasis. The present study was approved by the Institutional Ethics Review Board of the Catholic University of Korea (KC11RISIo887) and was in compliance with the Declaration of Helsinki.

\section{TACE procedure}

Using the selective/superselective technique, the tumor-feeding arteries were catheterized using a high- 
ly flexible coaxial microcatheter passed through a 5-Fr Yashiro catheter (Terumo, Tokyo, Japan), which was previously placed approximately in the hepatic artery. Specifically, for selective TACE, the tip of the microcatheter was placed into the hepatic arterial branch afferent to the segment in which the tumor was located. In the case of superselective TACE, the tip of the catheter was further advanced into the subsegmental branches feeding the tumor.

After microcatheter placement, a mixture of doxorubicin $\left(50 \mathrm{mg} / \mathrm{m}^{2}\right)$ and lipiodol (5 to $10 \mathrm{~mL}$ ) was injected under fluoroscopic control, followed by embolization with embolic materials such as gelform or polyvinyl alcohol particles until complete stasis was achieved in the segmental or subsegmental arterial branches in the tumor-feeding vessels. In the case of a parasitic tumor blood supply through accessory arteries (e.g., the inferior phrenic, internal mammary, or intercostal arteries), the patient underwent additional superselective treatment. Repeat treatment was scheduled within 2 weeks after follow-up imaging if residual viable tumor was detected.

\section{RFA procedure}

RFA was performed using a 17-gauge, internally cooled electrode with a 2 or $3 \mathrm{~cm}$ exposed tip (Cool-tip RF Ablation System, Valleylab, Boulder, CO, USA). After administration of analgesia and local anesthesia, the electrode needles were introduced into the tumor under ultrasonographic guidance, and RFA was performed according to the standard technique. The treatment response was evaluated based on subsequent dynamic CT, and additional RFA was performed until the tumor was completely ablated. When used in combination with TACE, RFA was performed within 4 weeks after TACE.

\section{Assessment and follow-up}

The treatment response was evaluated at 3 months (i.e., 1 month after the second treatment course) using the modified Response Evaluation Criteria in Solid Tumors. In the modified criteria of HCC tumor response, CR is the disappearance of any intratumoral arterial enhancement in all lesions; partial response (PR) is at least a 30\% decrease in the sum of the diameters of viable (contrast enhancement in the arterial phase) lesions; progressive disease (PD) is an increase of at least $20 \%$ in the sum of the diameters of viable lesions; stable disease is any case that does not qualify as either PR or PD [23]. Local recurrence was defined as an enhancement reappearing within the treated zone or $\leq 2.0 \mathrm{~cm}$ from its margins. When recurrence was recognized, patients were treated by RFA or TACE.

During the treatment period, laboratory tests, including serum $\alpha$-fetoprotein (AFP), albumin, bilirubin, AST, ALT and prothrombin time, and a liver dynamic CT (non-enhanced, arterial, portal, and delayed venous phase), were performed every 4 weeks after treatment. After achieving CR, patients were followed up every 3 months during the first year and every 3 to 6 months thereafter using laboratory tests and liver dynamic CT. Patients were followed up until loss of follow-up or death. The median follow-up period was 33.3 months (range, 6.8 to 80.9).

\section{Statistical analysis}

The primary endpoint was OS rate measured from the date of HCC diagnosis until death or the date of the last follow-up. The secondary endpoint was time to recurrence, which was measured from the date of CR to the date of local tumor recurrence or new lesions. All of the statistical tests, performed using SAS version 9.2 (SAS Institute Inc., Cary, NC, USA), were two-sided, and $p$ values $<0.05$ were considered statistically significant. Continuous variables are presented as median (range) and compared using Kruskal-Wallis tests. Categorical variables are presented as frequency (\%) and were compared using Fisher exact tests. The cumulative survival rates in each treatment group were estimated using the Kaplan-Meier method and log-rank test. To compare long-term outcomes of three treatments, hazard ratios (HRs) and confidence intervals (CIs) were calculated for each factor using univariate and multivariate Cox proportional hazards model adjusted for seven covariates.

To reduce the effect of potential confounding in an observational study, we also performed rigorous adjustment for differences in baseline characteristics of patients using propensity score methods [24]. We estimated propensity scores for each treatment for all of the patients using a non-parsimonious multiple logistic regression model. The model covariates included age, gender, cause of disease, tumor maximal diameter, tumor number, Child-Pugh Class, and level of AFP in the 
Table 1. Baseline characteristics of the hepatocellular carcinoma patients

\begin{tabular}{|c|c|c|c|c|}
\hline Characteristic & TACE + RFA $(n=87)$ & $\operatorname{TACE}(\mathrm{n}=71)$ & $\mathrm{RFA}(\mathrm{n}=43)$ & $p$ value \\
\hline Age, yr & $60.4(29.1-78.0)$ & $60.0(23.0-87.2)$ & $62.0(35.0-88.0)$ & 0.452 \\
\hline Sex & & & & 0.534 \\
\hline Male & $70(80.5)$ & $53(74.6)$ & $31(72.1)$ & \\
\hline Female & $17(19.5)$ & $18(25 \cdot 4)$ & $12(27 \cdot 9)$ & \\
\hline Etiology & & & & 0.959 \\
\hline Hepatitis B virus & $5^{8}(66.7)$ & $49(69.0)$ & $28(65.1)$ & \\
\hline Hepatitis $\mathrm{C}$ virus & $21(24 \cdot 1)$ & $14(19.8)$ & $9(20.9)$ & \\
\hline Alcohol & $5(5 \cdot 7)$ & $4(5.6)$ & $3(7 \cdot 0)$ & \\
\hline Other & $3(3 \cdot 5)$ & $4(5.6)$ & $3(7.0)$ & \\
\hline Laboratory test & & & & 0.128 \\
\hline Platelet count, $\times 10^{3} / \mathrm{mm}^{3}$ & $136 \pm 120$ & $110 \pm 50$ & $114 \pm 57$ & \\
\hline Total bilirubin, mg/dL & $1.2 \pm 1.9$ & $1.0 \pm 0.6$ & $1.27 \pm 1.4$ & \\
\hline Albumin, g/dL & $3.8 \pm 0.6$ & $3.7 \pm 0.5$ & $3.5 \pm 0.6$ & \\
\hline PT INR & $1.2 \pm 0.4$ & $1.2 \pm 0.5$ & $1.0 \pm 0.2$ & \\
\hline Tumor maximal diameters, $\mathrm{cm}$ & $2.5(1.0-4.6)$ & $2.5(1.0-4.7)$ & $2.2(1.3-4.7)$ & 0.223 \\
\hline$<3.0$ & $64(73.6)$ & $44(62.0)$ & $33(76.7)$ & 0.162 \\
\hline$\geq 3.0$ & $23(26.4)$ & $27(38.0)$ & $10(23 \cdot 3)$ & \\
\hline Tumor number & & & & 0.083 \\
\hline 1 & $64(73.6)$ & $41(57.7)$ & $35(81.4)$ & \\
\hline 2 & $17(19 \cdot 5)$ & $20(28.2)$ & $6(14.0)$ & \\
\hline 3 & $6(6.9)$ & $10(14.1)$ & $2(4.6)$ & \\
\hline Child-Pugh Class & & & & 0.165 \\
\hline A & $80(92.0)$ & $68(95.8)$ & $37(86.0)$ & \\
\hline $\mathrm{B}$ & $7(8.0)$ & $3(4.2)$ & $6(14.0)$ & \\
\hline Serum AFP, ng/dL & $18.7(1.5-4,190.0)$ & $24.9(1.7-25,714.0)$ & $14.4(1.9-1,210.0)$ & 0.264 \\
\hline$<20$ & $24(55.8)$ & $32(45 \cdot 1)$ & $46(52.9)$ & 0.469 \\
\hline$\geq 20$ & $19(44.2)$ & $39(54.9)$ & $41(47.1)$ & \\
\hline
\end{tabular}

Values are presented as median (range) or number (\%).

TACE, transarterial chemoembolization; RFA, radiofrequency ablation; PT, prothrombin time; INR, international normalized ratio; AFP, $\alpha$-fetoprotein.

serum. Each model between two treatments was well calibrated with good discrimination. For the inverse probability of treatment weights (IPTW) [25], the weights for patients who underwent one treatment were inverse of (1-propensity score), and weights for patients who the other were the inverse of propensity score. We finally compared outcomes (recurrence, local recurrence, and survival rates) after each treatment using weighted Cox proportional hazards regression model with the IPTW.

\section{RESULTS}

\section{Clinical characteristics of HCC patients}

The clinical characteristics of the 201 patients are shown in Table 1. This study included 154 males and 47 females. The median age was 60.4 years (range, 29.1 to 78.0 ) in the combination treatment (TACE + RFA) group, and 60.0 years (range, 23.0 to 87.2) and 62.0 years (range, 35.0 to 88.0) in the TACE and RFA groups, respectively. Male gender and hepatitis B virus infection were predominant in each group. More than $90 \%$ of patients had Child-Pugh class A liver function. A total of 201 patients 


\section{KJIM'}

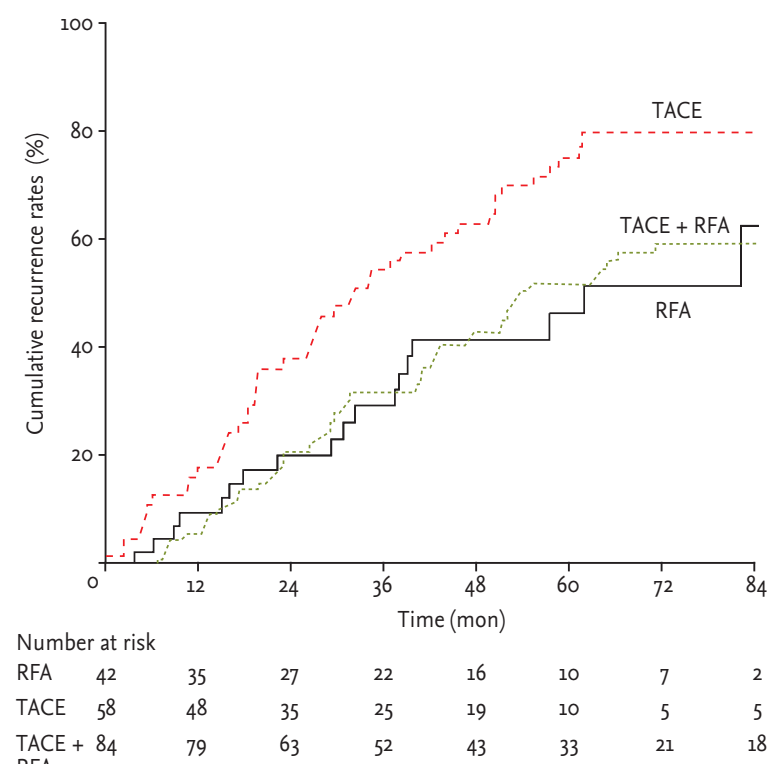

Figure 1. Recurrence rates. The recurrence rates in the transarterial chemoembolization (TACE) + radiofrequency ablation (RFA) and RFA groups were significantly lower than those in the TACE group $(p=0.015$ and $p=0.005$, respectively). Recurrence rates were not significantly different between the combination treatment and RFA groups $(p=0.776)$.

were diagnosed with early-stage HCC according to the BCLC staging classification. The tumor diameter was not significantly different among the three groups. The mean maximal tumor size was $2.5 \mathrm{~cm}$ (range, 1.0 to 4.6 ) in the combination treatment group, and $2.5 \mathrm{~cm}$ (range, 1.0 to 4.7 ) and $2.2 \mathrm{~cm}$ (range, 1.3 to 4.7 ) in the TACE and RFA groups, respectively. Patient demographics and tumor burden were not significantly different among the groups.

\section{Treatment response}

In total, 58 patients in the TACE group (81.6\%), 42 patients in the RFA group (97.6\%), and 84 patients in the TACE + RFA group (96.5\%) achieved CR at the time of response assessment (rate difference: TACE + RFA vs. TACE, 4.929 [ $95 \%$ CI, 1.305 to $18.622 ; p=0.019$ ] and TACE + RFA vs. RFA, 0.869 [95\% CI, 0.085 to $8.848 ; p=0.906]$ ). In addition, 10 patients in the TACE group, one patient in the RFA group, and three patients in the combination treatment group achieved PR.

\section{Recurrence}

During follow-up, HCC recurrence was identified in

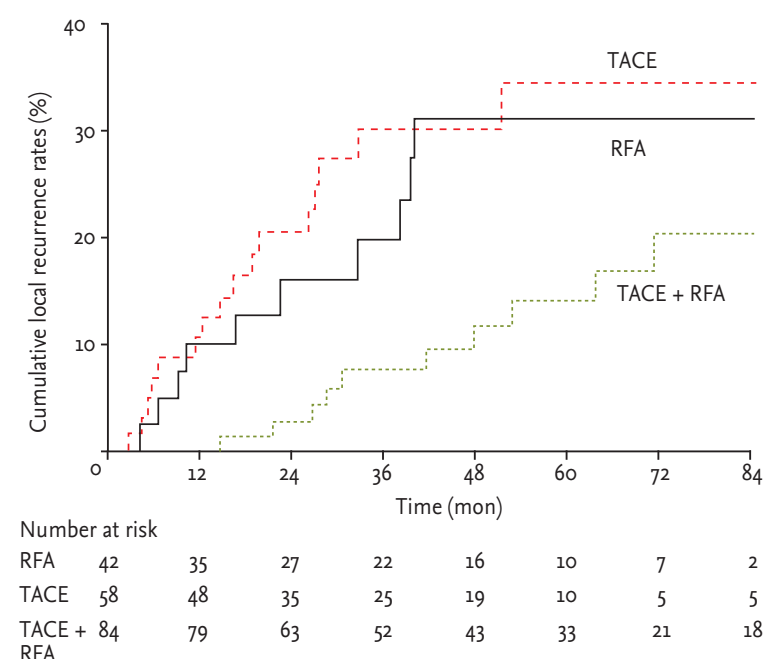

Figure 2. Local recurrence rates. In the transarterial chemoembolization (TACE) + radiofrequency ablation (RFA) group, the local recurrence rates were significantly lower than those in RFA group ( $p=0.014$ ); however, no significant difference in recurrence rates between RFA and TACE groups was observed $(p=0.787)$.

53 of 84 patients $(63.1 \%)$ in the combination treatment group, and in 48 of $58(82.7 \%)$ and 18 of 42 patients ( $42.9 \%)$ in the TACE and RFA monotherapy groups, respectively. The median time to recurrence was 19.5 months (range, 16.2 to 22.8). The median time to recurrence of patients in the combination treatment group, RFA group, and TACE group was 52.3, 61.7, and 31.5 months, respectively. The recurrence rate curve is shown in Fig. 1. The probabilities of recurrence rates at 1,3 , and 5 years in the combination treatment group $(6 \%, 33 \%$, and $54 \%$, respectively) and in the RFA group (10\%, $31 \%$, and $48 \%$, respectively) were significantly lower than those in the TACE group ( $17 \%, 58 \%$, and $78 \%$, respectively; $p=0.015$ and $p=0.005$, respectively). No significant difference in recurrence rates was found between the combination treatment and RFA groups $(p=0.776)$. Subgroup analysis according to local tumor recurrence showed that in the combination treatment group, the local recurrence rates were significantly lower than those in the RFA and TACE groups ( $p=0.014$ and $p=0.019$, respectively), whereas the recurrence rates in the RFA and TACE groups were not significantly different $(p=0.787$ ) (Fig. 2 ).

In a multivariate analysis of recurrence rates using the multivariate-adjusted and IPTW, the combination treatment group showed significantly lower local recurrence rates than the RFA and TACE monotherapy groups (HR, 
Table 2. Comparison of recurrence and local recurrence in each treatment group

\begin{tabular}{|c|c|c|c|c|c|c|c|c|}
\hline \multirow[t]{2}{*}{ Treatment } & \multirow[t]{2}{*}{ No } & \multirow[t]{2}{*}{ Yes } & \multicolumn{3}{|c|}{ Multivariate-adjusted } & \multicolumn{3}{|c|}{$\begin{array}{c}\text { Adjusted by inverse probability } \\
\text { of treatment weights }\end{array}$} \\
\hline & & & HR & $95 \%$ CI & $p$ value & HR & $95 \% \mathrm{CI}$ & $p$ value \\
\hline \multicolumn{9}{|l|}{ Recurrence } \\
\hline \multicolumn{9}{|c|}{ RFA vs. TACE + RFA } \\
\hline RFA & $24(57.1)$ & $18(42.9)$ & 1.000 & - & 0.776 & 1.000 & - & 0.563 \\
\hline TACE + RFA & $31(36.9)$ & $53(63.1)$ & 1.089 & $0.605-1.962$ & - & 0.866 & $0.533-1.409$ & - \\
\hline \multicolumn{9}{|c|}{ TACE vs. TACE + RFA } \\
\hline TACE & $10(17 \cdot 3)$ & $48(82.7)$ & 1.000 & - & 0.015 & 1.000 & - & 0.009 \\
\hline TACE + RFA & $31(36.9)$ & $53(63.1)$ & 0.581 & $0.374-0.901$ & - & 0.594 & $0.403-0.876$ & - \\
\hline \multicolumn{9}{|l|}{ RFA vs. TACE } \\
\hline RFA & $24(57.1)$ & $18(42.9)$ & 1.000 & - & 0.005 & 1.000 & - & 0.047 \\
\hline TACE & $10(17 \cdot 3)$ & $48(82.7)$ & 2.543 & $1.322-4.895$ & - & 1.671 & $1.006-2.775$ & - \\
\hline \multicolumn{9}{|l|}{ Local recurrence } \\
\hline \multicolumn{9}{|c|}{ RFA vs. TACE + RFA } \\
\hline RFA & $32(76.1)$ & $10(23.9)$ & 1.000 & - & 0.014 & 1.000 & - & 0.008 \\
\hline TACE + RFA & $74(88.1)$ & $10(11.9)$ & 0.299 & $0.114-0.785$ & - & 0.309 & $0.130-0.736$ & - \\
\hline \multicolumn{9}{|c|}{ TACE vs. TACE + RFA } \\
\hline TACE & $42(72.9)$ & $16(27.1)$ & 1.000 & - & 0.019 & 1.000 & - & 0.011 \\
\hline TACE + RFA & $74(88.1)$ & $10(11.9)$ & 0.354 & $0.149-0.840$ & - & 0.352 & $0.158-0.787$ & - \\
\hline \multicolumn{9}{|l|}{ RFA vs. TACE } \\
\hline RFA & $32(76.1)$ & $10(23.9)$ & 1.000 & - & 0.787 & 1.000 & - & 0.872 \\
\hline TACE & $42(72.9)$ & $16(27.1)$ & 0.880 & $0.347-2.229$ & - & 0.939 & $0.435^{-2.025}$ & - \\
\hline
\end{tabular}

Values are presented as number (\%). Adjusted for age, gender, cause, tumor size, tumor number, child class, $\alpha$-fetoprotein. HR, hazard ratio; CI, confidence interval; RFA, radiofrequency ablation; TACE, transarterial chemoembolization.

$0.309 ; 95 \%$ CI, 0.130 to $0.736 ; p=0.008$ and HR, $0.352 ; 95 \%$ CI, 0.158 to $0.787 ; p=0.011$, respectively) (Table 2). Conversely, total recurrence rates including local recurrence and the appearance of a new HCC were not different between the TACE + RFA and RFA groups (HR, 0.866; 95\% CI, 0.533 to $1.409 ; p=0.563$ ) (Table 2 ).

\section{OS rates}

The median follow-up period was 33.3 months (range, 3.8 to 80.9). During follow-up, 14 of 87 patients (17.2\%) in the combination treatment group, 19 of 71 patients (26.7\%) in the TACE group, and 11 of 43 patients $(25.5 \%)$ in the RFA group died. The major causes of death were tumor progression (43.1\%), liver dysfunction due to complications of cirrhosis (liver failure, hepatorenal syndrome, and rupture of esophageal varices; $47.7 \%$ ) and infection (9.0\%). There was no death related to treatment.

The median survival times in the TACE + RFA group and TACE and RFA groups were 80.1, 65.2, and 48 months, respectively. The probabilities of OS at 1,3 , and 5 years were $98 \%, 95 \%$, and $90 \%$ in the TACE + RFA group; $98 \%, 90 \%$, and $83 \%$ in the TACE group; and $94 \%, 84 \%$, and $71 \%$ in the RFA group, respectively. The combination treatment group had significantly better long-term survival rates than the RFA group, and showed a trend favoring an OS benefit compared to the TACE group; however, significant differences between the RFA and TACE groups were not observed $(p=0.044, p=0.046$, and $p=0.771$, respectively, based on log-rank test $)$ (Fig. 3).

We analyzed the survival rates of each treatment modality according to tumor size. In tumors $<3 \mathrm{~cm}$ in size, the survival rate probabilities at 1,3 , and 5 years in the combination treatment group (98\%, $95 \%$, and $90 \%$, respectively) were significantly higher than those in the TACE group (98\%, 90\%, and 85\%, respectively) and in the RFA group (94\%, 82\%, and 71\%, respectively; $p=0.017$ 
Table 3. Comparison of the overall survival rates in each treatment group

\begin{tabular}{|c|c|c|c|c|c|c|c|c|}
\hline \multirow{2}{*}{ Treatment } & \multicolumn{2}{|c|}{ Overall survival } & \multicolumn{3}{|c|}{ Multivariate-adjusted } & \multicolumn{3}{|c|}{$\begin{array}{c}\text { Adjusted by inverse probability } \\
\text { of treatment weights }\end{array}$} \\
\hline & Alive & Death & HR & $95 \% \mathrm{CI}$ & $p$ value & HR & $95 \% \mathrm{CI}$ & $p$ value \\
\hline \multicolumn{9}{|c|}{ RFA vs. TACE + RFA } \\
\hline RFA & $32(74.4)$ & $11(25 \cdot 6)$ & 1.000 & & 0.044 & 1.000 & & 0.041 \\
\hline TACE + RFA & $73(83.9)$ & $14(16.1)$ & 0.371 & $0.141-0.973$ & & 0.422 & $0.185-0.964$ & \\
\hline \multicolumn{9}{|c|}{ TACE vs. TACE + RFA } \\
\hline TACE & $52(73.2)$ & $19(26.8)$ & 1.000 & & 0.046 & 1.000 & & 0.124 \\
\hline TACE + RFA & $73(83.9)$ & $14(16.1)$ & 0.476 & $0.230-0.986$ & & 0.588 & $0.299-1.156$ & \\
\hline \multicolumn{9}{|l|}{ RFA vs. TACE } \\
\hline RFA & $32(74.4)$ & $11(25.6)$ & 1.000 & & 0.771 & 1.000 & & 0.550 \\
\hline TACE & $52(73.2)$ & $19(26.8)$ & 1.138 & $0.476-2.721$ & & 0.797 & $0.379-1.677$ & \\
\hline
\end{tabular}

Values are presented as number (\%). Adjusted for age, gender, cause, tumor size, tumor number, child class, $\alpha$-fetoprotein. HR, hazard ratio; CI, confidence interval; RFA, radiofrequency ablation; TACE, transarterial chemoembolization.

and $p=0.004$, respectively). Significant differences between the RFA and TACE groups were not observed $(p=$ 0.315) (Fig. 4A). However, in larger tumors $\geq 3 \mathrm{~cm}$ in size, the survival rate probabilities at 1,3 , and 5 years in the combination treatment group (98\%, $84 \%$, and $82 \%$, respectively) were not significantly lower than those in the TACE (98\%, 86\%, and 80\%, respectively; $p=0.952$ ) and RFA groups (98\%, 84\%, and 64\%, respectively; $p=0.763$ ). Significant differences between the RFA and TACE groups were not observed $(p=0.702)$ (Fig. $4 \mathrm{~B}$ ).

We adjusted the prognostic factors that affected the OS rates. The variables included age, gender, etiology, tumor size, tumor single/multiple tumor, Child-Pugh class, and serum AFP level. In a multivariate analysis of OS using the Cox regression model with the IPTW, combination treatment showed significant survival benefit compared to RFA (HR, 0.422; 95\% CI, 0.185 to 0.964 ; $p=0.041$ ) (Table 3). These results suggest that TACE + RFA combination treatment yields better OS rates in early-stage HCC patients.

\section{Complications}

Common complications in all of the groups were fever, pain, and increased levels of AST, ALT, or total bilirubin. Serious complications including tumor seeding after RFA, liver failure, or treatment-related death were not observed after treatment.

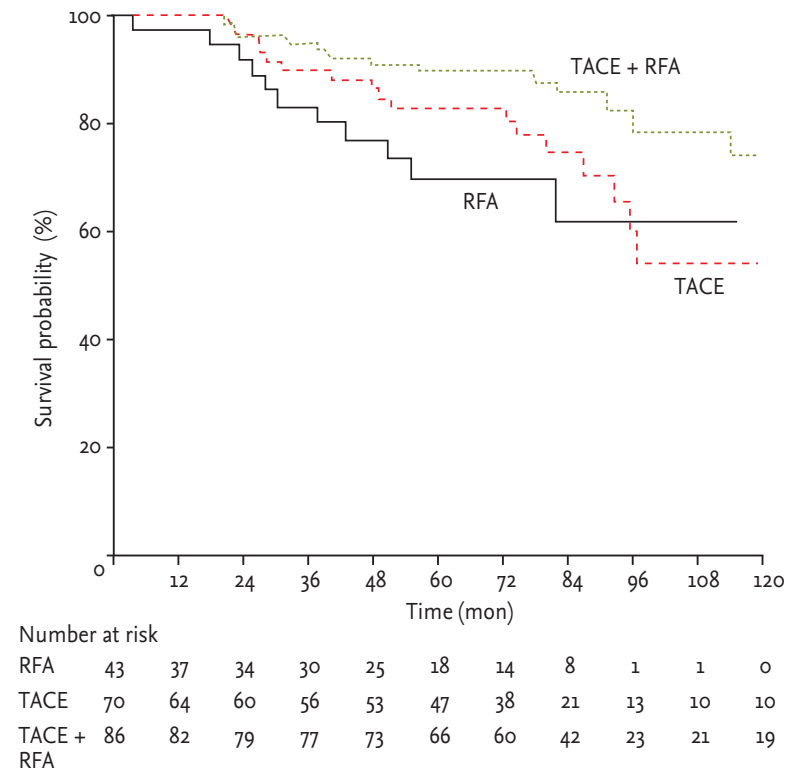

Figure 3. Cumulative curve of overall survival plotted based on the Kaplan-Meier method and compared using the logrank test. Patients in the transarterial chemoembolization (TACE) + radiofrequency ablation (RFA) group showed better overall survival rates than those in the RFA or TACE groups ( $p=0.044$ and $p=0.046$, respectively).

\section{DISCUSSION}

Both RFA and TACE $\mathrm{m}$ onotherapies beneficially affect survival and have been established as valuable treatment modalities for patients with unresectable HCC. Recent studies have suggested that RFA + TACE combination 

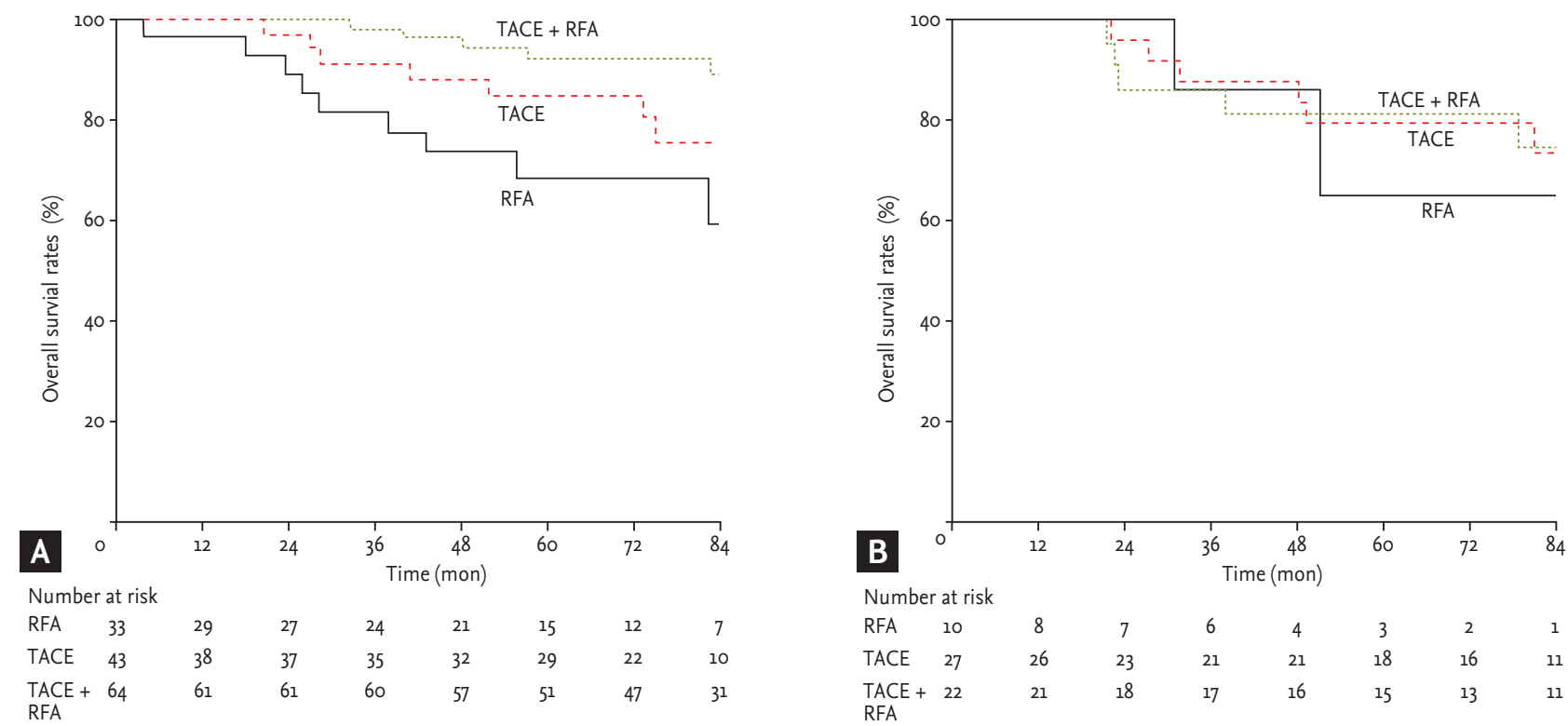

Figure 4. Subgroup analysis of the overall survival of hepatocellular carcinoma patients undergoing transarterial chemoembolization (TACE) + radiofrequency ablation (RFA), RFA, or TACE according to the main tumor size. (A) There were significant differences in the long-term survival of patients undergoing TACE + RFA in tumors $<3 \mathrm{~cm}$ in size compared to patients receiving only RFA or TACE treatment $(p=0.017$ and $p=0.004$, respectively). (B) Significant differences were not observed in the overall survival benefit of the patients undergoing TACE + RFA in tumors $\geq 3 \mathrm{~cm}$ in size compared to patients receiving only RFA or TACE treatment ( $p=0.763$ and $p=0.952$, respectively).

therapy could be expanded to patients with resectable HCC and transplant candidates [26,27]. According to BCLC staging and treatment algorithm, RFA is considered curative for early-stage HCC, and may be comparable to surgical resection in terms of OS and recurrence $[26,28]$. However, this technique has limitations. As tumor size increases, the complete necrosis caused by ablation is generally reduced due to increased blood flow, thereby leading to heat loss. Reportedly, the rate of complete necrosis according to pathological analysis after RFA was approximately $50 \%$, but was $61 \%$ to $63 \%$ in nodules $<3 \mathrm{~cm}$ in size and $15 \%$ in HCCs 3 to $5 \mathrm{~cm}$ in size [29].

TACE represents another treatment option for early-stage HCC due to various factors, including tumor location and the presence of comorbidities or patients rejecting surgery. TACE has also shown long-term survival rates for HCC patients within the Milan criteria, similar to RFA [30]. However, to date, limited data are available concerning its efficacy in inducing extensive tumor necrosis in early-stage HCC, and it remains controversial. Larger HCCs are fed by larger feeding arteries, thereby leading to greater hypervascularity of the nodule during angiography, whereas smaller nodules tend to be more hypovascular. Thus, TACE was less efficient in treating smaller nodules, which may limit its use to only larger lesions.

Hypothetically, performing TACE prior to RFA (TACE + RFA) could reduce heat loss. As a result, the ablation volume could be increased, allowing greater treatment response in cases of larger HCCs than with RFA or TACE alone. In a recent study, HCCs 3 to $5 \mathrm{~cm}$ in size had a higher rate of necrosis than smaller tumors after superselective/selective TACE, supporting the use of combination treatment involving RFA and superselective/ selective TACE in early-stage HCC [20]. Furthermore, TACE can treat undetected satellite lesions outside the RFA-induced necrosis zone. A randomized controlled trial is usually the standard method for comparing various treatment modalities. However, due to current HCC guidelines, which suggest that early-stage HCC patients should consider RFA rather than TACE as the first-line treatment, conducting a randomized, head-to-head comparison is difficult. We investigated the treatment response, recurrence rates, and OS benefit of TACE + RFA combination therapy in patients with early-stage 
HCC using a propensity score model.

In this study, the CR rates at 3 months were $96.5 \%$, $97.6 \%$, and $81.6 \%$ in the combination treatment, RFA group, and TACE group, respectively. Although the CR rate in the TACE group was greater than that obtained in previous studies due to the superselective/selective approach, the treatment responses in the groups that included RFA were significantly better than those with TACE in early-stage HCC. These data suggest that TACE is limited as a palliative treatment compared to RFA.

The recurrence rates at 1,3 , and 5 years in patients with early-stage HCC in the TACE + RFA and RFA groups were comparable (TACE + RFA: 6\%, 33\%, and 54\%; RFA: $10 \%, 31 \%$, and $48 \%$, respectively). In contrast, the TACE group had significantly higher recurrence rates $(17 \%$, $58 \%$, and $78 \%$, respectively). Therefore, the treatment groups that included RFA showed lower recurrence rates than TACE, as well as a better treatment response. In subgroup analysis of local recurrence, TACE + RFA showed better local control than RFA (11.9\% and $23.3 \%$, $p=0.008)$. Local recurrence rates in the RFA group were comparable to those previously reported ( $2.4 \%$ to $30 \%$ ) $[5,28,31,32]$. However, in our study, local recurrence rate was not different between the RFA and TACE groups. A probable reason is that RFA often causes vaporization of intracellular water and formation of microbubbles within the ablation zone; the resulting high intralesional pressure may facilitate tumor dissemination along the portal venous system within the same segment as RFA, thus accounting for early local recurrence [33]. This result may have contributed to the lack of difference in local recurrence rates of both groups in the propensity mode. This indicates that TACE + RFA combination treatment reduces microscopic local tumor recurrence more effectively than RFA or TACE monotherapies.

Furthermore, combination treatment significantly outperformed RFA in terms of OS. The better local control of disease recurrence following TACE + RFA combination treatment may benefit survival by delaying the deleterious effects of tumor progression. Importantly, the propensity score analysis consistently showed that combination treatment was superior to RFA in terms of prolonged survival. However, there was no difference in survival benefit between the TACE + RFA and TACE groups in our study $(p=0.124)$, although recurrence rates were significantly lower in the combination treatment group than in the TACE group $(p=0.124$ and $p=0.009$, respectively). Our results showed that TACE + RFA led to better OS than TACE based on the adjusted Cox model. However, TACE + RFA was not an independent favorable prognostic factor in the propensity mode, suggesting that other factors may play more critical roles in determining OS. In addition, this was probably due to the immediate treatment of local and new tumor progression using appropriate modalities, including repeat RFA or TACE in both groups. Subgroup analysis showed that among patients with a tumor $<3 \mathrm{~cm}$ in size, the TACE + RFA group had significantly better long-term survival than the TACE or RFA groups $(p=0.017$ and $p=0.004$, respectively). Therefore, patients with BCLC stage A HCC may survive longer after treatment with TACE + RFA than those who receive only RFA or TACE.

This study had a number of strengths. First, the treatment modality, including curative treatment such as RFA, showed a better response than superselective/selective TACE. Second, the combination treatment yielded more effective control of local tumor recurrence than RFA or TACE. Finally, this comparative study showed an improvement in OS benefit using TACE + RFA combination treatment, particularly in tumors sized $<3 \mathrm{~cm}$. A propensity score analysis is a useful strategy for minimizing selection bias when the impact of different treatments on recurrence and survival are interrupted in an observational study.

This study had several limitations. First, it was retrospective in design and conducted in a single center. A randomized controlled trial would be ideal but difficult because in clinical practice, factors such as tumor location and patient status affect treatment decisions. Second, the group of patients receiving RFA therapy was relatively small. Specifically, performing RFA is difficult due to current clinical practice for early HCC patients with tumor $\geq 3 \mathrm{~cm}$ in size. Therefore, determining the significant difference in OS in tumors $\geq 3 \mathrm{~cm}$ in size may be difficult.

In conclusion, we showed that TACE + RFA is an effective treatment for early-stage HCC for lower local tumor recurrence and leads to better survival rates compared to RFA or TACE alone, particularly for tumors $<3 \mathrm{~cm}$ in size. Additional studies are needed for the selection of suitable HCC patients for TACE + RFA combination treatment. 


\section{KEY MESSAGE}

1. Combining radiofrequency ablation (RFA) with transarterial chemoembolization (TACE) to treat hepatocellular carcinoma may have a synergistic effect.

2. In this study, the treatment efficacy and safety of combination treatment was better than RFA or TACE monotherapy.

3. TACE + RFA combination treatment should be considered in suitable early-stage hepatocellular carcinoma patients.

\section{Conflict of interest}

No potential conflict of interest relevant to this article was reported.

\section{Acknowledgments}

This work was supported by a National R\&D Program grant for cancer control, Ministry of Health, Welfare and Family Affairs, Republic of Korea (Ro620390-1).

\section{REFERENCES}

1. Parkin DM, Bray F, Ferlay J, Pisani P. Estimating the world cancer burden: Globocan 2000. Int J Cancer 2001;94:153-156.

2. El-Serag HB, Mason AC. Rising incidence of hepatocellular carcinoma in the United States. N Engl J Med 1999;340:745-750.

3. Bruix J, Sherman M, Llovet JM, et al. Conclusions of the Barcelona-2000 EASL conference: European Association for the Study of the Liver. J Hepatol 2001;35:421-430.

4. Lencioni RA, Allgaier HP, Cioni D, et al. Small hepatocellular carcinoma in cirrhosis: randomized comparison of radio-frequency thermal ablation versus percutaneous ethanol injection. Radiology 2003;228:235-240.

5. Lin SM, Lin CJ, Lin CC, Hsu CW, Chen YC. Radiofrequency ablation improves prognosis compared with ethanol injection for hepatocellular carcinoma $<$ or $=4 \mathrm{~cm}$. Gastroenterology 2004;127:1714-1723.

6. Kagawa T, Koizumi J, Kojima S, et al. Transcatheter arterial chemoembolization plus radiofrequency ablation therapy for early stage hepatocellular carcinoma: comparison with surgical resection. Cancer 2010;116:3638-3644.
7. Choi D, Lim HK, Rhim H, et al. Percutaneous radiofrequency ablation for early-stage hepatocellular carcinoma as a first-line treatment: long-term results and prognostic factors in a large single-institution series. Eur Radiol 2007;17:684-692.

8. Livraghi T, Meloni F, Di Stasi M, et al. Sustained complete response and complications rates after radiofrequency ablation of very early hepatocellular carcinoma in cirrhosis: is resection still the treatment of choice? Hepatology 2008;47:82-89.

9. N'Kontchou G, Mahamoudi A, Aout M, et al. Radiofrequency ablation of hepatocellular carcinoma: long-term results and prognostic factors in 235 Western patients with cirrhosis. Hepatology 2009;50:1475-1483.

10. Bruix J, Sherman M; American Association for the Study of Liver Diseases. Management of hepatocellular carcinoma: an update. Hepatology 2011;53:1020-1022.

11. Llovet JM, Bruix J. Systematic review of randomized trials for unresectable hepatocellular carcinoma: chemoembolization improves survival. Hepatology 2003;37:429-442.

12. Livraghi T, Goldberg SN, Lazzaroni S, Meloni F, Solbiati L, Gazelle GS. Small hepatocellular carcinoma: treatment with radio-frequency ablation versus ethanol injection. Radiology 1999;210:655-661.

13. McGhana JP, Dodd GD 3rd. Radiofrequency ablation of the liver: current status. AJR Am J Roentgenol 2001;176:316.

14. Livraghi T, Goldberg SN, Lazzaroni S, et al. Hepatocellular carcinoma: radio-frequency ablation of medium and large lesions. Radiology 2000;214:761-768.

15. Dupuy DE, Goldberg SN. Image-guided radiofrequency tumor ablation: challenges and opportunities: part II. J Vasc Interv Radiol 2001;12:1135-1148.

16. Curley SA. Radiofrequency ablation of malignant liver tumors. Ann Surg Oncol 2003;10:338-347.

17. Llovet JM, Di Bisceglie AM, Bruix J, et al. Design and endpoints of clinical trials in hepatocellular carcinoma. J Natl Cancer Inst 2008;100:698-711.

18. Bruix J, Sala M, Llovet JM. Chemoembolization for hepatocellular carcinoma. Gastroenterology 2004;127(5 Suppl 1):S179-S188.

19. Lencioni R. Loco-regional treatment of hepatocellular carcinoma. Hepatology 2010;52:762-773.

20. Golfieri R, Cappelli A, Cucchetti A, et al. Efficacy of selective transarterial chemoembolization in inducing tumor necrosis in small $(<5 \mathrm{~cm})$ hepatocellular carcinomas. 
Hepatology 2011;53:1580-1589.

21. Akamatsu M, Yoshida H, Obi S, et al. Evaluation of transcatheter arterial embolization prior to percutaneous tumor ablation in patients with hepatocellular carcinoma: a randomized controlled trial. Liver Int 2004;24:625-629.

22. Marelli L, Stigliano R, Triantos C, et al. Treatment outcomes for hepatocellular carcinoma using chemoembolization in combination with other therapies. Cancer Treat Rev 2006;32:594-606.

23. Lencioni R, Llovet JM. Modified RECIST (mRECIST) assessment for hepatocellular carcinoma. Semin Liver Dis 2010;30:52-60.

24. D'Agostino RB Jr. Propensity score methods for bias reduction in the comparison of a treatment to a non-randomized control group. Stat Med 1998;17:2265-2281.

25. Robins JM, Hernan MA, Brumback B. Marginal structural models and causal inference in epidemiology. Epidemiology 2000;11:550-560.

26. Sohn MH, Jeong YJ, Jeong HJ, et al. F-18 FDG PET/CT findings of huge cholesterol granuloma in multiple ribs. Clin Nucl Med 2011;36:942-944.

27. Ravaioli M, Grazi GL, Piscaglia F, et al. Liver transplantation for hepatocellular carcinoma: results of down-staging in patients initially outside the Milan selection criteria. Am J Transplant 2008;8:2547-2557.
28. Chen MS, Li JQ, Zheng Y, et al. A prospective randomized trial comparing percutaneous local ablative therapy and partial hepatectomy for small hepatocellular carcinoma. Ann Surg 2006;243:321-328.

29. Pompili M, Mirante VG, Rondinara G, et al. Percutaneous ablation procedures in cirrhotic patients with hepatocellular carcinoma submitted to liver transplantation: assessment of efficacy at explant analysis and of safety for tumor recurrence. Liver Transpl 2005;11:1117-1126.

30. Hsu CY, Huang YH, Chiou YY, et al. Comparison of radiofrequency ablation and transarterial chemoembolization for hepatocellular carcinoma within the Milan criteria: a propensity score analysis. Liver Transpl 2011;17:556566.

31. Camma C, Di Marco V, Orlando A, et al. Treatment of hepatocellular carcinoma in compensated cirrhosis with radio-frequency thermal ablation (RFTA): a prospective study. J Hepatol 2005;42:535-540.

32. Tateishi R, Shiina S, Teratani T, et al. Percutaneous radiofrequency ablation for hepatocellular carcinoma: an analysis of 1000 cases. Cancer 2005;103:1201-1209.

33. Ng KK, Poon RT, Lo CM, Yuen J, Tso WK, Fan ST. Analysis of recurrence pattern and its influence on survival outcome after radiofrequency ablation of hepatocellular carcinoma. J Gastrointest Surg 2008;12:183-191. 